International Journal of Computer Networks \& Communications (IJCNC) Vol.10, No.4, July 2018

\title{
AN EFFICIENT BANDWIDTH OPTIMIZATION AND MINIMIZING ENERGY CONSUMPTION UTILIZING EFFICIENT RELIABILITY AND INTERVAL DisCREPANT Routing (ERIDR) Algorithm
}

\author{
Sivashanmugam. $\mathrm{N}^{1}$ and Jothi Venkateshwaran. $\mathrm{C}^{2}$ \\ ${ }^{1}$ Research Scholar, PG \& Research Department of Computer Science, Presidency \\ College, Madras University, Chennai-60005, Tamilnadu, India \\ ${ }^{2}$ Professor \& Head, Dept. of Computer Science and Engineering, St. Peter's Institute of \\ Higher Education and Research, Chennai, Tamilnadu, India
}

\begin{abstract}
The bandwidth utilization plays a vital role in a Wireless Sensor Network (WSN) that transmits data packets from source peer to perspective destination peer without any packet loss and time delay. In a conventional system, two main features cannot be satisfied concurrently such as low delay and high data reliability and then the peer was transferred fewer data packets and it optimized with regular bandwidth rate. Moreover, the convention of bandwidth in network routers influences the quality of service $(Q o S)$. To overcome the above issues, an Efficient Reliability and Interval Discrepant Routing (ERIDR) algorithm is proposed to optimize bandwidth utilization on the router network with the help of bandwidth optimizer. The bandwidth optimizer allocates required bandwidth for data transmission to each peer simultaneously to ensure the bandwidth efficiency. The proposed design is to optimize bandwidth utilization of every peer and increase data processing via higher bandwidth rate that reduces time delay and minimizes energy consumption. The proposed method establishes a high bandwidth rate router to transmit data concurrently from source peer to destination peer (peer-to-peer) without any packet loss by initializing host IP address for every peer. Based on Experimental evaluations, proposed methodology reduces 3.32 AD (Average Delay), 0.05 ET (Execution Time), 5.44 EC (Energy Consumption) and 0.28 BU (Bandwidth Utilization) compared than existing methodologies.
\end{abstract}

\section{KEYWORDS}

Bandwidth Optimization, Energy Consumption (EC), Efficient Reliability and Interval Discrepant Routing (ERIDR) Algorithm, quality of service (QoS), Average Delay (AD), Execution Time (ET), and Bandwidth Utilization $(B U)$.

\section{INTRODUCTION}

Wireless networking communications evolved in our environment widely through various technologies for data transmission from one place to another with bandwidth capacity through channel mediums. The bandwidth utilization is a key concept that transmits data in bits/second, the rate of bandwidth speed differs (kbps or Mbps) according to the size of data packets and to control the data transceiver channels for quality throughout. Internet protocols are the emerging routing network for transmission and receiving of data packets with the help of sliceable optical transponder (SOT) carries multiple data flows concurrently. Data packet contains control information and payload of the user during transmission. Cognitive radio networks found an optimal route that increased bandwidth utilization on the selected path for following paired network devices and throughput performance was a highly efficient range of (20 to $40 \mathrm{Mbps}$ ) strength in the selected optimal path. In the channel, bandwidth efficiency is necessary to 
communicate router peers who carry the volume of data packets simultaneously without any modification via congested traffic and transmit faster for further re-transmission if any packet loss.

The fundamental issues in the existing system are a delay, energy consumption, data reliability and bandwidth utilization. Information Communication and Technology (ICT) industries consuming more energy that outlets carbon dioxide emissions, a large amount of bandwidth utilization occur on the network. The high-performance computers (HPC) consumes Mega-Watts of electricity every day for processing data packets from one peer to another where multiple cooling systems are required to cool down HPC. Un-usable bandwidth wasted in the network itself where it is not shared or used among another peer which carries a huge amount of load and consume too much of energy as well as bandwidth. The complexity is data congestion in network traffic due to low bandwidth rate for a large amount of data packet transmission.

The proposed system implements an Efficient Reliability and Interval Discrepant Routing (ERIDR) algorithm optimizes un-usable bandwidth on the network and minimizes the bandwidth capacity according to the size of data packets transmitted. Modules consist of Source Peer (SP), Network Router (NR), Destination Peer (DP), and Bandwidth Optimizer (BO). Source peer selects the file from the perspective directories, initializing each peer to further transmission and proving destination address for packet delivery. Network Router (NR) transmitting data through peer for minimal bandwidth utilization, less energy consumption and processing quicker for reducing time delay and increasing execution time. Destination Peer (DP) receives the data packets from routers efficiently. Bandwidth Optimizer is initialized to monitor transmission and calculates the amount of bandwidth utilized by each peer; the un-usable bandwidth is later shared among another peer, or bandwidth rate for the following peer is reduced. The contribution of this paper follows:

- To minimize bandwidth utilization during the data transmission of the packets through peers.

- To avoid un-usable bandwidth on the routing network.

- To transmit the data packet with minimal time delay and less energy consumption.

- To monitor a routing process of the data packets in a faster execution standard.

- To reduce the Average Delay, Execution Time, Energy Consumption and Bandwidth Utilization compare than existing approaches.

The rest of the paper contribution is followed by section 2 illustrates the related work which expresses the closest technology of the proposed methodology. Section 3 introduces the proposed methodology, design, implementations steps with proposed algorithms. Section 4 discusses the implemented result and comparison analysis. Section 5 concludes the overall work with future outcomes.

\section{RELATED WORK}

Guo et al. [1] discussed an extended SDN controller, and it enhanced the light-path bandwidth consumption. It combined numerous fine-granularity streams into a light-path, through an objective of optimizing bandwidth utilization while guaranteed broadcast performances. However, an extended SDN controller does not offer bandwidth resource management. Zhou et al. [2] described an iterative sub-cluster partition algorithm utilized to share data among mobile devices in a coverage range by utilizing base stations and device-to-device communication clusters. D2D communication clusters performed retransmission process simultaneously if any packet loss occurs on the cluster. However, retransmission time is high. Zhu et al. [3] developed the integration of RMSA (routing, modulation, and spectrum assignment) and Hybrid Single/Multi-Path Routing (HSMR) algorithm and it analyzed the path carries data from one peer to another for data transmission. The disadvantages of the designed algorithm utilized multiple hops 
which transfer in the same path. Han et al. [4] designed a Green-energy Aware Latency-Aware (GALA) algorithm which reduced power consumptions on Macro base-stations (MBS) and Pico base-stations (PBS) coverage areas. The MBS \& PBS technologies are converted to green which controls power consumption on the grid. In the algorithm, energy was not stable and reliable due to traffic congestion.

Barshan et al. [5] introduced an efficient architecture with the integration of Customer premises equipment (CPE) and Network Function Virtualization (NFV). It utilized to transfer data packets to the external network which increased the flexibility of data transmission, energy efficiency framework and it offered clients better commodity services. It requires changing the functions of aspects according to the users when the architecture was deployed. Guo et al. [6] suggested a scheme Fair Network Bandwidth Allocation (FALLOC) and it offered VM services to clients in effective base bandwidth utilized a distributed algorithm which managed un-usable bandwidth on the network. It shared with other VM that needs high bandwidth rate with the help of falloc. However, the falloc scheme does not accomplish the conventional TCP (Transmission Control Protocol). Barakat et al. [7] described an off-path cache method, and it solved content cache over the network. It increased the performance in egress, and it was a global hit ratio on multiple routers. The method reduced bandwidth usage and improvised the capacity of cache since the method changes router allocation for every deployment process. Wang et al. [8] designed an energy-aware routing algorithm which reduced power consumption in the software-defined network. In network topology, the six-node topology initialized with 400 nodes to process requests more than capable of nodes initialized and it reduced processing time. The disadvantage of the routing algorithm will not save energy and data consistency at the similar time. Bradai et al. [9] illustrated a cellular software-defined network (CSDN), and it improved the performance of data reliability, bandwidth availability. CSDN controller managed server authentication, accounting, security and third-party application from LTE Network. However, the CSDN architecture was inadequate for mobile networks.

Chai et al. [10] investigated RRM (Radio Resource Management) algorithm over congestion traffic network utilized dynamic QoS-based bandwidth allocation framework which monitored the existing and new connection automatically. It assigned bandwidth based on their service and increased the capacity of transmission. Reddy et al. [11] developed an Ad-hoc On Demand Vector (AODV) routing protocol, and it identified complex migration during data transmission and associates waiting for a queue of packets. It computed available bandwidth which increased the performance of the system. Bartelt et al. [12] evaluated the Macro-base station and Pico-base station power consumption, migration cost, congestion networks where interference of coverage range was solved by providing Joint and Optimization algorithm for managing bandwidth utilization among those stations in a particular range. Felemban et al. [13] implemented MMSPEED routing protocol for data reliability and reduced time consumption during data transmission from the service provider to destination peer without any packet loss; the proposed algorithm ensures QoS. Wang et al. [14] expressed multi-hop nodes around source node which reduced the energy consumption of router nodes as well as time taken for reaching the destination (Sink) through Pair-Wise Directional Geographical Routing algorithm that monitored surrounding nodes power consumption and data transmission rate concurrently. In [15] developed an efficient cluster-based multipath routing method which every cluster includes two cluster heads, one to gather cluster information and the other to broadcast information. 


\section{SYSTEM METHODOLOGY}

In the section explained the system architecture with proposed techniques and algorithm details. The proposed system architecture is explained in Figure 1. The main objective of the proposed system improves the data reliability and delay distinguished services (RDDS) simultaneously, and a bandwidth optimizer allocates required bandwidth for data transmission of every peer concurrently to ensure bandwidth efficiency. An Efficient Reliability and Interval Discrepant Routing (ERIDR) algorithm is proposed to reduce the amount of un-usable bandwidth of each peer. It establishes high bandwidth rate router to transmit data concurrently from source to destination (peer-to-peer) without any packet loss by initializing host IP address for every peer. It enhances the throughput and minimizes the energy, delay and bandwidth utilization.

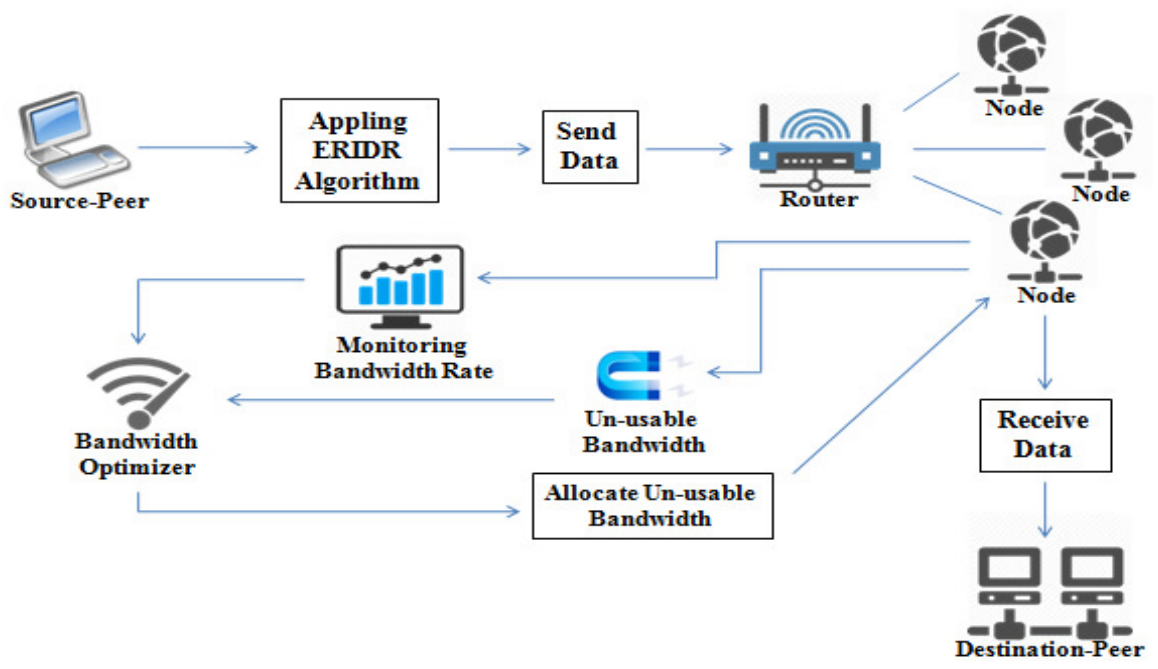

Figure.1 Architecture Diagram of ERIDR Algorithm

\subsection{Source Peer}

The source peer selects the content file, initialize the routers and after that transmit to the particular destination peer. The source peer will transfer the selected content file to the router, and then the router selects the minimum distance path and transmits to the particular destination peer.

\subsection{Router}

The router controls the various networks to offer data storage service. In network n-number of peers are presented $(\mathrm{p} 1, \mathrm{p} 2, \mathrm{p} 3, \mathrm{p} 4, \mathrm{p} 5 \ldots)$. In a router source, the peer can provide analysis peer details and attacked peers. The source peer will transmit their content file to router and router will select minimum distance path and transmits to the particular destination peer. If a few attackers are investigated in a peer after that, the router will connect to another peer and transmit particular client.

\subsection{Obtrusion Discovery Framework (ODF) Controller}

The ODF controller contains the two stages. If reliability or malicious content occurs in router after that ODF controller is enabled. In the first stage, the DNS packets, clear stream, fine-grained ODF user discovery and traffic filter are present. The main objective is detecting all peers within the observed network that connect in ODF communications. To investigate raw traffic gathered at the edge of the observed network and apply a pre-filtering stage to reject network streams that are unlikely to be produced by ODF applications. Then investigate the remaining traffic and extract 
some statistical aspects of recognizing streams produced by ODF users. In the second stage, Coarse-grained ODF reliability or malicious content discovery, Fine-grained ODF client discovery and Reliability or Malicious content are present. In the system investigates the traffic produced by the ODF users and categorizes them into each legitimate ODF users or ODF reliability or malicious content.

\subsection{Destination Peer}

The destination peer can receive the content file from the router. The source peer will transmit the content file to the router, and then the router will transfer to the particular destination peer. The destination peer receives the content file without modifying the file contents. The clients may receive particular content files within the network only.

\subsection{Bandwidth optimizer}

In the module, bandwidth optimizer will manage bandwidth rate among other peers. If peer carries fewer amounts of data packets, and the peer bandwidth is shared with another peer who carries a huge amount of data packets.

\subsection{Efficient Reliability and Interval Discrepant Routing (ERIDR) Algorithm}

An Efficient Reliability and Interval Discrepant Routing (ERIDR) algorithm is reducing the amount of un-usable bandwidth of each peer. Four features are affecting the peer-to-peer delay such as transfer interval, the competition of the radio channel, queuing interval, and path length. Transfer interval is limited and it associated with bandwidth. Competition of radio channel is particularly based on the contention of MAC; a packet has to compete for the channel access and wait for content transfer until the channel is idle. Queuing delay is a huge queue seriously takes the delay of transfer of the packets. Generally, the path length has additional hops for packet transmission; the huge possible delay will suffer. The physical constraint establishes the transfer interval, and the MAC influences the competition of radio channel. The ERIDR algorithm aims to minimize the transfer interval, packet loss, bandwidth utilization, energy and improves the throughput. Consider the network with different high reliability or delay content files. Let $\mathrm{c}$ be the identifier of various applications. The main process of ERIDR algorithm at peer works as follows. First, if the queue at peer is not empty, then bandwidth is computed for the packet at the head of the queue. Calculate the bandwidth and select the next hop. The peer sends the content file to the destination peer. ERIDR build up a potential field along with the bandwidth and queue length data to investigate the under-utilized paths. The packets with huge reliability requirements will be forwarded to the next hop with lesser queue length. Every application allotted a packet size, and it describes the degree sensitivity to the delay.

ERIDR describes a Maximum Update Interval (MUI) and a Least Update Interval (LUI) among two successive modify data. MUI is always larger than LUI. The modified data should be transfer among an LUI and MUI at least once. If no data file is received from a neighbour through two MUIs intervals, in the neighbour will be measured dead, and ERIDR will recompute the bandwidth and other related values. A modify data will be transfer. If MUI timer expires the time will be elapsed because transferring the last modified data exceeds the MUI; a new modify data will be transferred immediately no matter whether the bandwidth or queue length has changed. The queue length variation exceeds a certain threshold. If the queue length of a peer has varied compared with last successful modified data and the elapsed time exceeds the LUI because the last modify data. If the bandwidth size of a peer has modified and the elapsed time exceeds the LUI because the last successful modify data. The pseudo code of proposed algorithm is explained below. 
Input: Text Types of Input Data

Output: Display the Average Delay (AD), Execution Time (ET), Energy Consumption (EC) and Bandwidth Utilization (BU).

\section{Procedure:}

Start

Arrange all the peers (source peer, router, and destination peer)

Browse any data file to send destination peer;

Select destination peer;

Apply ERIDR algorithm;

Initialize peer

If compute optimization of bandwidth

Measure un-usage bandwidth during data transmission

Compute optimization of bandwidth

Else

Bandwidth waste

\section{End if}

Identify the shortest path for data transmission process

If send data

Transmit data to destination peer;

Calculate \& visualize AD, EC, ET, and BU;

Else

Failed to transmit the data

Fail to compute $\mathrm{AD}, \mathrm{EC}, \mathrm{ET}$, and $\mathrm{BU}$;

End

\section{RESUlt AND DisCUSSION}

\subsection{Implementation Setup}

The proposed techniques are deployed with Intel i6 Core processor, with 16 GB RAM, 60 GB Memory with Windows7 Ultimate operating systems. The algorithm is implemented in Java programming environment by using NetBeans 8.0.2, MYSQL database 5.5, Java Remote Access Library with 50 peers in a centralized server environment. The proposed algorithm is computed with following constraints namely Average Delay, Execution Time, Throughput and Bandwidth Utilizations performance evaluation metrics.

\subsection{Performance Matrix}

The proposed methodology is computed on different kinds of constraints to calculate the efficiency of techniques. The proposed technique is highly dedicated to providing efficient content data file transmission with minimal resource optimization on similar QoS guarantee services. 


\subsubsection{Average Delay (AD)}

The average delay is described as the time difference between the current data packets received time, and the previous data packets received time. It exhibits data transmission delay between source peer and destination peers. The average delay is computed in Equation (1).

$$
A D=\frac{\text { Pkt RecvdTime }- \text { Pkt Sent Time }}{\text { time }}
$$

Where Pkt is packets and Recvd is Received packet.

\subsubsection{Throughput}

Throughput is the average ratio of successful data delivered to the destination peer. The throughput specifies efficiency of the proposed method. The throughput is calculated in Equation (2).

$$
\text { Throughput }=\frac{\sum_{0}^{n} \text { Pkts Received }(n) * \text { Pkt Size }}{1000}
$$

\subsubsection{Energy Consumption}

The amount of energy remaining in a node at the current instance of time is called as residual energy. The difference between the initial energy and the residual energy over the simulation time provides the rate at which energy is consumed by the network operations. The energy consumption is computed in Equation (3) and (4).

$$
\begin{gathered}
\text { Energy } T_{x}=\frac{(330 X \text { data size })}{(2 \times 106)} \\
\text { Energy } R_{x}=\frac{(230 \times \text { data size })}{(2 \times 106)}
\end{gathered}
$$

Where $R_{x}$ is Residual Energy and $T_{x}$ is Initial Energy.

\subsubsection{Bandwidth Utilization}

The bandwidth utilization is computed on each peer require file transmission size \& system efficiency. The bandwidth ensures the how techniques effective to avoid traffic and congestion issues with minimal physical resource utilization. The Bandwidth Utilization is estimated in equation (5).

$$
B W_{\text {utilization }}=\frac{B W_{\text {Assigned }}}{N p r}
$$

Where, $\mathrm{BW}_{\text {Assigned }}$ is bandwidth allocation of a peer in-network for data transmissions. Npr indicates the total number of peer request for efficient data transmissions in networks. 


\subsubsection{Execution Time}

The execution is defined as the total time to transmit the data source peer to destination peer. The execution time is computed in equation (6).

$$
\text { Execution Time }=\text { FS X CPFS X T }
$$

Where CPFS- cycles per file size, FS- File Size and T is time.

Tables (eg, Table 1) explain the Average Delay (AD), Energy Consumption (EC), Execution Time (ET) and Bandwidth Utilizations (BU) for respective input parameters with existing methods. Tables (eg, Table 1) displays the average value on all respective evaluation matrix \& input parameters with Adhoc On-Demand Distance Vector (AODV) [16], Joint Routing, Dynamic Source Routing Protocol (DSR) [16] and Dynamic Manet On-demand Routing Protocol (DYMO) [16] existing methods. Along with Tables (eg, Table 1), it noticed that Efficient Reliability and Interval Discrepant Routing (ERIDR) algorithm performs well on all evaluation matrix and Input parameters compare than existing methods.

Table 1. Average Delay, Energy Consumption, Execution Time, Bandwidth Utilization for Dynamic Routing.

\begin{tabular}{|c|c|c|c|c|}
\hline Algorithm & $\begin{array}{c}\text { Average } \\
\text { Delay (s) }\end{array}$ & $\begin{array}{c}\text { Execution } \\
\text { Time (s) }\end{array}$ & $\begin{array}{c}\text { Energy } \\
\text { Consumption } \\
\text { (Joules) }\end{array}$ & $\begin{array}{c}\text { Bandwidth } \\
\text { Utilization } \\
\text { (Mbps) }\end{array}$ \\
\hline AODV & 7.58 & 0.17 & 58.55 & 3.78 \\
\hline DSR & 24.3 & 0.16 & 53.18 & 2.65 \\
\hline DYMO & 4.68 & 0.15 & 52.89 & 1.63 \\
\hline ERIDR & 1.36 & 0.10 & 47.45 & 1.35 \\
\hline
\end{tabular}

Figures (eg, Figure 2) demonstrate the average delay in seconds, and the proposed algorithm ERIDR is comparing existing algorithms such as AODV, DSR, and DYMO. The processing of data packets from the source peer to destination peer utilizing ERIDR algorithm produced low average delay when it compared to existing methodologies. The DSR method provides the high average delay for transmitting data packets from the source and destination. DYMO algorithm is the closest existing algorithm compare than the proposed algorithm.

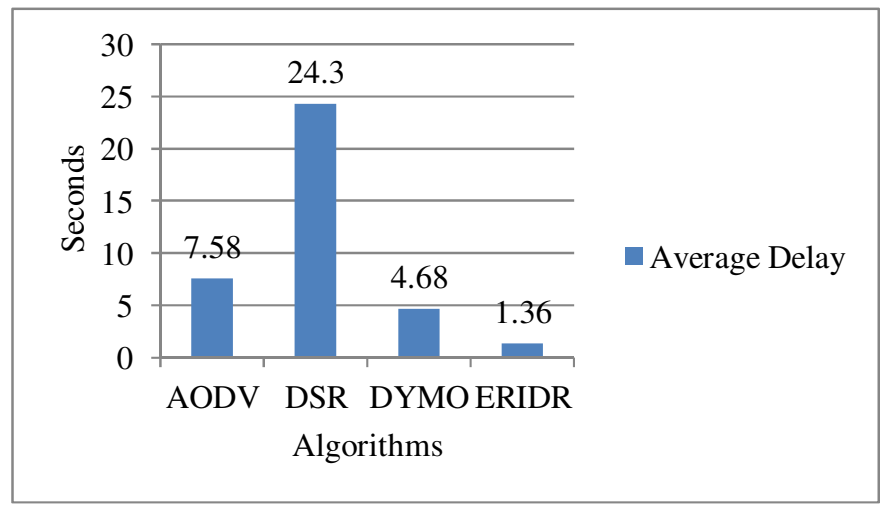

Fig. 2 Average Delay of Dynamic Routing 
Figures (eg, Figure 3) demonstrate the throughput in kbps, and the proposed algorithm ERIDR is comparing existing algorithms such as AODV, DSR, and DYMO. The processing of data packets from the source peer to destination peer utilizing ERIDR algorithm produces less execution time when it compared to existing methodologies. The AODV algorithm provides high execution time for transmitting data packets from the source and destination. DYMO algorithm is the nearest existing algorithm to compare than proposed algorithm.

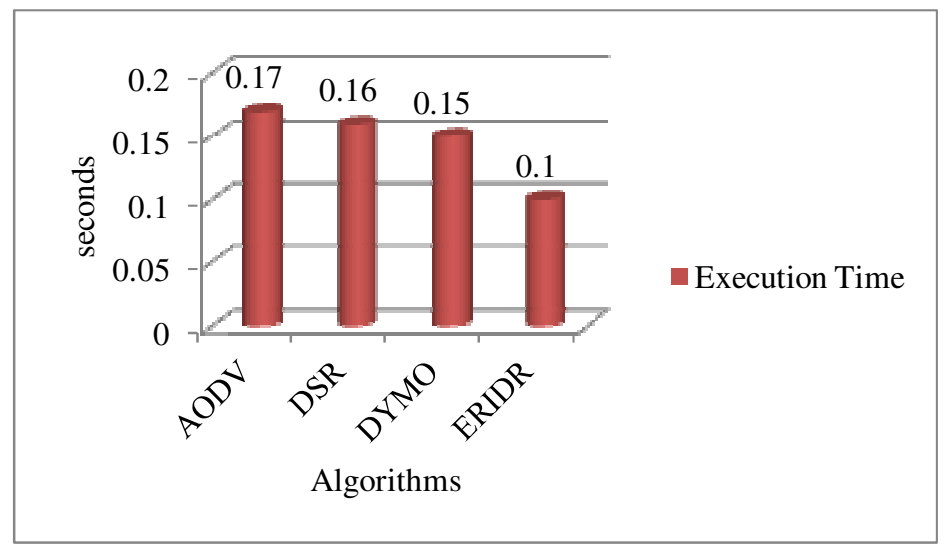

Fig. 3 Execution Time of Dynamic Routing

Figures (eg, Figure 4) demonstrate the energy consumption in joules, and the proposed algorithm ERIDR is comparing existing algorithms such as AODV, DSR, and DYMO. The processing of data packets from the source peer to destination peer utilizing ERIDR algorithm consumed low energy when it compared to existing methodologies. The AODV algorithm is consuming more energy for transmitting data packets from the source and destination peer. DYMO algorithm is the nearest existing algorithm to compare than proposed algorithm.

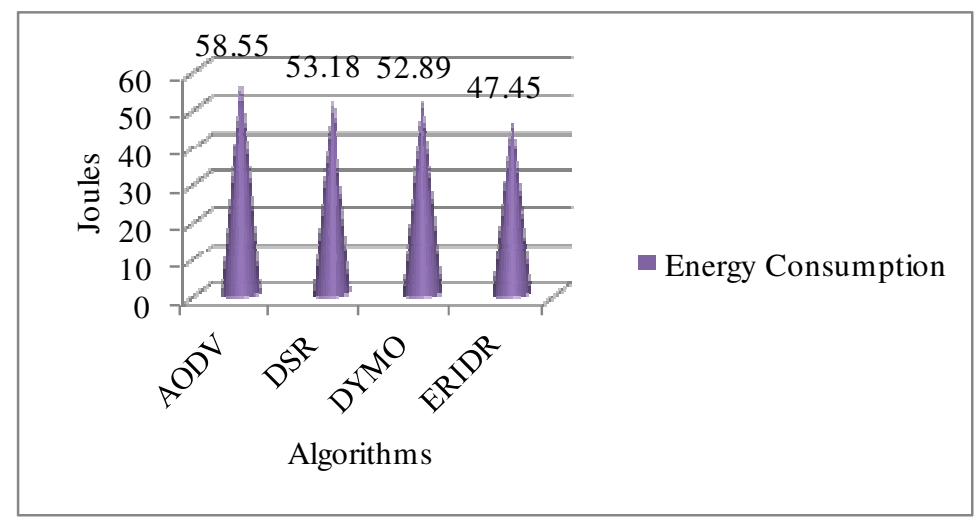

Fig. 4 Energy consumption of Dynamic Routing

Figures (eg, Figure 5) demonstrate the encryption time in milliseconds, and the proposed algorithm ERIDR is comparing existing algorithms such as AODV, DSR, and DYMO. The processing of data packets from the source peer to destination peer utilizing ERIDR algorithm consumes less bandwidth when it compared to existing methodologies. The AODV algorithm is utilizing more bandwidth for transmitting data packets from the source and destination peer. DYMO algorithm is the nearest existing algorithm to compare than proposed algorithm. 


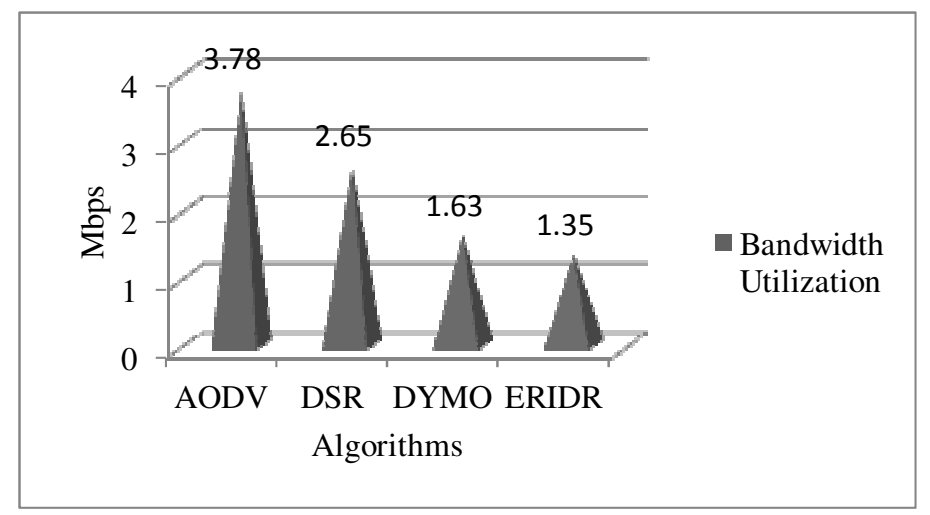

Fig. 5 Bandwidth Utilization of Dynamic Routing

Based on Figures (eg, Figure 2,3,4,\& 5), it is observed that, the proposed ERIDR technique computes average delay, execution time, energy consumption and bandwidth utilization for finding the efficiency. The proposed ERIDR is evaluated with AODV, DSR and DYMO existing techniques on behalf of average delay, execution time, energy consumption and bandwidth utilization. The AODV is utilized to determine multiple paths between the source peer and the destination peer in every route determines. However, it is the high overhead of data transmission and failed to maintain complete path details a t every peer. The proposed ERIDR approach is less overhead and effectively maintains complete path details. The DSR is an efficient source routing the method, when the total number of peers is less it transmits data to source peer to destination peer effectively. However, the number of peers is increased; the data transmission is failed due to more traffic in the network. The proposed ERIDR method is utilized to transmit data effectively for high number of peers. The proposed technique closest competitor is DYMO on overall parameters. DYMO techniques improved route maintenance and route discovery. It improved the performance of various commercials application for data transmissions. However, DYMO technique failed to support asymmetric links. Proposed method improves the bandwidth utilization of un-usage bandwidth. Proposed ERIDR reduces 3.32 AD (Average Delay), 0.05 ET (Execution Time), 5.44 EC (Energy Consumption) and 0.28 BU (Bandwidth Utilization). Finally, the paper claims the proposed ERIDR algorithm performs best on every evaluation matrix \& respective input parameters.

\section{Conclusions}

The An Efficient Reliability and Interval Discrepant Routing (ERIDR) algorithm utilized to minimize some quality of service metrics such as bandwidth utilization and energy consumption. The main concept of a proposed system to optimize bandwidth utilization on router network with the help of the bandwidth optimizer that allocates required bandwidth for data transmission of each node simultaneously to ensure bandwidth efficiency. It optimizes bandwidth utilization of every node and increases data processing via higher bandwidth rate that reduces time delay and minimizes energy consumption. It establishes high bandwidth rate router to transmit data concurrently from source to destination (peer-to-peer) without any packet loss by initializing host IP address for every node. Proposed ERIDR reduces 3.32 AD (Average Delay), 0.05 ET (Execution Time), 5.44 EC (Energy Consumption) and 0.28 BU (Bandwidth Utilization). Finally, the paper claims the proposed ERIDR methodology performs best on every evaluation matrix \& respective input parameters.

\section{ACKNOWLEDGMENTS}

The authors would like to thank everyone, just everyone! 
International Journal of Computer Networks \& Communications (IJCNC) Vol.10, No.4, July 2018

\section{REFERENCES}

[1] Guo, W., Bin, W., Jin, Y., Hu, W., \& Xia, M., (2014) "Joint optimization of transmission performance and bandwidth utilization based on software-defined network," In Optical Fiber Communication Conference, Optical Society of America, pp. W4H-2.

[2] Zhou, B., Hu, H., Huang, S. Q., \& Chen, H. H., (2013) "Intracluster device-to-device relay algorithm with optimal resource utilization," IEEE transactions on vehicular technology, Vol. 62, No. 5, pp. 2315-2326.

[3] Zhu, Z., Lu, W., Zhang, L., \& Ansari, N., (2013) "Dynamic service provisioning in elastic optical networks with hybrid single-/multi-path routing," Journal of Lightwave Technology, Vol. 31, No. 1, pp. 15-22.

[4] Han, T., \& Ansari, N., (2013) "Green-energy aware and latency aware user associations in heterogeneous cellular networks," In Globecom Workshops (GC Wkshps), pp. 4946-4951.

[5] Barshan, M., Moens, H., Famaey, J., \& De Turck, F., (2015) "Algorithms for advance bandwidth reservation in media production networks," In Integrated Network Management (IM), 2015 IFIP/IEEE International Symposium, pp. 183-190.

[6] Guo, J., Liu, F., Tang, H., Lian, Y., Jin, H., \& Lui, J. C., (2013) "Falloc: Fair network bandwidth allocation in iaas datacenters via a bargaining game approach", In Network Protocols (ICNP), 2013 21st IEEE International Conference, pp. 1-10.

[7] Barakat, C., Kalla, A., Saucez, D., \& Turletti, T., (2013) "Minimizing bandwidth on peering links with deflection in named data networking," In Communications and Information Technology (ICCIT), 2013 Third International Conference, pp. 88-92.

[8] Wang, R., Jiang, Z., Gao, S., Yang, W., Xia, Y., \& Zhu, M., (2014) "Energy-aware routing algorithms in software-defined networks", In World of Wireless, Mobile and Multimedia Networks (WoWMoM), 2014 IEEE 15th International Symposium, pp. 1-6.

[9] Bradai, A., Singh, K., Ahmed, T., \& Rasheed, T., (2015) "Cellular software-defined networking: a framework," IEEE communications magazine, Vol. 53, No. 6, pp. 36-43.

[10] Chai, R., Wang, X., Chen, Q., \& Svensson, T., (2013) "Utility-based bandwidth allocation algorithm for heterogeneous wireless networks," Science China Information Sciences, Vol. 56, No. 2, pp. 1-13.

[11] Reddy, C. P., Gopal, J., \& Sangaiah, A. K., (2014) "Efficient bandwidth utilization with congestion control for wireless mesh networks," Indian Journal of Science and Technology, Vol. 7, No. 11, pp. 1780-1787.

[12] Bartelt, J., Fehske, A., Klessig, H., Fettweis, G., \& Voigt, J., (2013) "Joint bandwidth allocation and small cell switching in heterogeneous networks," In Vehicular Technology Conference (VTC Fall), 2013 IEEE 78th, pp. 1-5.

[13] Felemban, E., Lee, C. G., Ekici, E., Boder, R., \& Vural, S., (2005) "Probabilistic QoS guarantee in reliability and timeliness domains in wireless sensor networks," In INFOCOM 2005. 24th Annual Joint Conference of the IEEE Computer and Communications Societies. Proceedings IEEE, Vol. 4, pp. 2646-2657.

[14] Wang, J., Zhang, Y., Wang, J., Ma, Y., \& Chen, M., (2015) "PWDGR: pair-wise directional geographical routing based on wireless sensor network," IEEE Internet of things journal, Vol. 2, No. 1, pp. 14-22.

[15] Anandan, R. K., \& Amirthalingam, S., (2016) "Cluster-based Multipath Routing for Wireless Multimedia Sensor Networks," International Journal of Intelligent Engineering and Systems, Vol. 9, No. 4, pp. 79-87.

[16] Chandane, M. M., Bhirud, S. G., \& Bonde, S. V., (2012) "Performance analysis of IEEE 802.15.4", International Journal of Computer Applications, pp. 0975-8887. 